\title{
Many-Body Quantum Dynamics of Initially Trapped Systems due to a Stark Potential: Thermalization versus Bloch Oscillations
}

\author{
Pedro Ribeiro $\oplus^{1,2,3,{ }^{*}}$ Achilleas Lazarides, ${ }^{3,4}$ and Masudul Haque ${ }^{3,5}$ \\ ${ }^{1}$ CeFEMA, Instituto Superior Técnico, Universidade de Lisboa, Avenida Rovisco Pais, 1049-001 Lisboa, Portugal \\ ${ }^{2}$ Beijing Computational Science Research Center, Beijing 100193, China \\ ${ }^{3}$ Max Planck Institute for the Physics of Complex Systems, Nothnitzer Strasse 38, 01187 Dresden, Germany \\ ${ }^{4}$ Interdisciplinary Centre for Mathematical Modelling and Department of Mathematical Sciences, Loughborough University, \\ Loughborough, Leicestershire LE11 3TU, United Kingdom \\ ${ }^{5}$ Department of Theoretical Physics, Maynooth University, Maynooth, County Kildare, Ireland
}

(Received 1 April 2019; revised manuscript received 13 January 2020; accepted 20 February 2020; published 17 March 2020)

\begin{abstract}
We analyze the dynamics of an initially trapped cloud of interacting quantum particles on a lattice under a linear (Stark) potential. We reveal a dichotomy: initially trapped interacting systems possess features typical of both many-body-localized and thermalizing systems. We consider both fermions $(t-V$ model $)$ and bosons (Bose-Hubbard model). For the zero and infinite interaction limits, both systems are integrable: we provide analytic solutions in terms of the moments of the initial cloud shape and clarify how the recurrent dynamics (many-body Bloch oscillations) depends on the initial state. Away from the integrable points, we identify and explain the timescale at which Bloch oscillations decohere.
\end{abstract}

DOI: 10.1103/PhysRevLett.124.110603

Introduction.-Historically, many-body quantum physics in solid-state systems focused on low-energy parts of the many-body spectrum. In contrast, in nuclear physics the full spectrum was considered important [1-3]. This perspective gained prominence in recent years, as experiments with cold atoms [4-7] have inspired the study of nonequilibrium situations in isolated quantum systems [8-11]. In an isolated situation, energy conservation ensures that a system with an initially high energy will never explore the low-energy parts of the spectrum. The quantum dynamics of isolated systems poses new challenging questions, such as whether observables thermalize [8-10,12].

A well-known example for which isolation leads to drastically different dynamics is the phenomenon of Bloch oscillations $[13,14]$. Particles in a tight-binding lattice subject to a linear potential, e.g., due to gravity or an electric field, do not accelerate toward lower potentials, but rather undergo local oscillations. For a single particle, the shape and/or position of the particle wave function oscillates, perfectly periodically [13-16]. Long after its prediction, Bloch oscillations were observed in semiconductor superlattices [17,18], in cold atoms [19-22], and in periodic photonic structures [23-25]. In cold atom experiments, Bloch oscillations have by now been observed many times

Published by the American Physical Society under the terms of the Creative Commons Attribution 4.0 International license. Further distribution of this work must maintain attribution to the author(s) and the published article's title, journal citation, and DOI. Open access publication funded by the Max Planck Society.
[5,19-22,26-43], and are used widely as a measurement tool, e.g., for metrological applications [29,31,35,37], to detect Dirac points [36], etc. Some experiments have also explored the effect of interparticle interactions on Bloch oscillations [26,28,32-34,39]. Theoretical treatments of Bloch oscillations have addressed a variety of single-particle situations [15,16,44-56], interacting few-particle systems [57-61], and interacting many-body systems [46,48,62-82]. Interactions have been treated both in mean-field (e.g., Gross-Pitaevskii) regimes $[46,48,64-69,73,77,82]$ and beyond the mean-field regime [62,70-72,74,79,81].

Recent experiments [39] have found, by tuning bosonic on-site repulsion, the collapse and revival of the oscillation of the cloud position, with the revival period proportional to interaction strength. In addition, sufficiently far from the noninteracting point, the atom cloud was found to have "chaotic" behavior leading to rapid relaxation.

In this work, we address the real-time dynamics of an initially trapped interacting lattice system subject to a linear potential. We present a comprehensive study for two representative systems (featuring bosons and fermions), for all interaction regimes. At zero or infinite interaction, each model becomes integrable. For intermediate interactions, away from integrability, we have an example of many-body localization without disorder [83-89], where nevertheless a version of thermalization is valid when we focus on the part of the Hilbert space spanned by states in which particles are confined within a connected spatial region, i.e., the subspace explored by initially trapped systems. We show that the dynamics within such a subspace is thermalizing. 
At the integrable points, the dynamics is perfectly periodic. We provide a series of exact analytical results for the cloud dynamics in these cases. For strongly interacting (hard-core) bosons, we show dynamical generation (and periodic disappearance) of fragmented condensation of an initial uncondensed cloud. At strong (weak) initial trapping, the dynamics consists primarily of width (position) oscillations. At intermediate trapping, the skewness undergoes unusual dynamics during every period, of which we do not know of an analog in the literature. Near the integrable points, we show and explain beating behavior of the cloud dynamics, with linear dependences on the integrability-breaking parameter. This explains and generalizes the experimental observation of Ref. [39].

Models.-We consider $N_{p}$ particles on an infinite lattice subjected to a tilt potential. The total Hamiltonian,

$$
H=\mathcal{T}+\mathcal{E}+\mathcal{V},
$$

consists of a kinetic term $\mathcal{T}=-J / 2 \sum_{j}\left(a_{j}^{\dagger} a_{j+1}+\right.$ H.c. $)$, with hopping amplitude $J$ and site index $j$, a potential term $\mathcal{E}=E \sum_{j} j a_{j}^{\dagger} a_{j}$ due to a constant tilt strength $E$, and an interaction term $\mathcal{V}$. We consider two families of models: the Bose-Hubbard model (BHM) for which $\mathcal{V}=U / 2 \sum_{j} b_{j}^{\dagger} b_{j}^{\dagger} b_{j} b_{j}$, in which case the particles are bosons $a_{j}=b_{j}$, and the $t-V$ model (Ft-VM) with $\mathcal{V}=V \sum_{j} c_{j}^{\dagger} c_{j+1}^{\dagger} c_{j+1} c_{j}$, featuring interacting spinless fermions $a_{j}=c_{j}$.

As initial state $\left|\Phi_{0}\right\rangle$, we will mostly use the ground state of the nontilted system in the presence of a harmonic potential $\left(H_{0}=\mathcal{T}+\mathcal{V}+W \sum_{j} j^{2} a_{j}^{\dagger} a_{j}\right)$, parametrized by the dimensionless constant $\tilde{\rho}=N_{p} \sqrt{W / J}[90,91]$. The initial condition can be varied from an extended Gaussianlike cloud (small $\tilde{\rho}$ ) to a highly packed state at large $\tilde{\rho}$. We also consider initial states which are product states, e.g., of the form $\left|\Phi_{0}\right\rangle=a_{i+1}^{\dagger} a_{i+2}^{\dagger} \ldots a_{i+N_{p}}^{\dagger}|0\rangle$. For bosons at $U=\infty$ and for fermions at all $V \neq \infty$, the ground state has this form at large $\tilde{\rho}$. The system undergoes dynamics under Hamiltonian (1) with this initial state (a "quantum quench").

In addition to $U, V=0$, in both strong interacting regimes $(U, V \rightarrow \infty)$, the dynamics is that of a set of noninteracting particles. For $U \rightarrow \infty$ (BHM), double occupancy is kinematically forbidden and the finite-energy Hilbert space reduces to that of hard-core bosons. In this limit the BHM maps to the Ft-VM with $V=0$ via a JordanWigner transformation. The spectrum of the Ft-VM with $V \rightarrow \infty$ and $L$ sites can also be shown to map onto that of a Ft-VM with $L-N_{p}$ sites and $V=0$ [92]. In all these (effectively) noninteracting cases, the spectrum of the tilted Hamiltonian consists of equally spaced highly degenerate levels, with spacing $E$. This yields periodic evolution, with period $T=2 \pi / E$, for any initial state. In fact, exact analytical solutions can be found for the many-body evolution [92]. Away from these "free" cases the dynamics is nonintegrable: either because the nontilted model is already so (BHM) or because a finite tilt breaks the integrability present in the $E=0$ case (Ft-VM).

Longtime behavior and thermalization.-Figures 1(a) and $1\left(\mathrm{a}^{\prime}\right)$ show the eigenenergies $\varepsilon_{\alpha}$ of $H$, corresponding to eigenvectors $|\alpha\rangle$, as a function of the interaction strength, color coded with $\left|c_{\alpha}\right|^{2}$, with $c_{\alpha}=\left\langle\alpha \mid \Phi_{0}\right\rangle$ the overlap amplitude with the initial state. Only some eigenstates have a non-negligible overlap with the initial state; the other eigenenergies are not visible. For fixed $N_{p}$, increasing the chain length $L$ (with fixed $N_{p}$ ) increases the Hilbert space dimension polynomially, rendering the spectrum dense at $L \rightarrow \infty$, but leaves Figs. 1(a) and 1(a') invariant. Density profiles of the many-body eigenstates which have non-negligible $\left|c_{\alpha}\right|^{2}$ are exponentially localized within a length proportional to $1 / E$. This can be traced to the fact that a cloud of atoms in an infinite system is always in the dilute density regime; as interations are short-range, if the cloud expands too much the particles cease to interact with each other. Thus the exponential localization of the singleparticle eigenstates $[13,14]$ ensures that the many-body

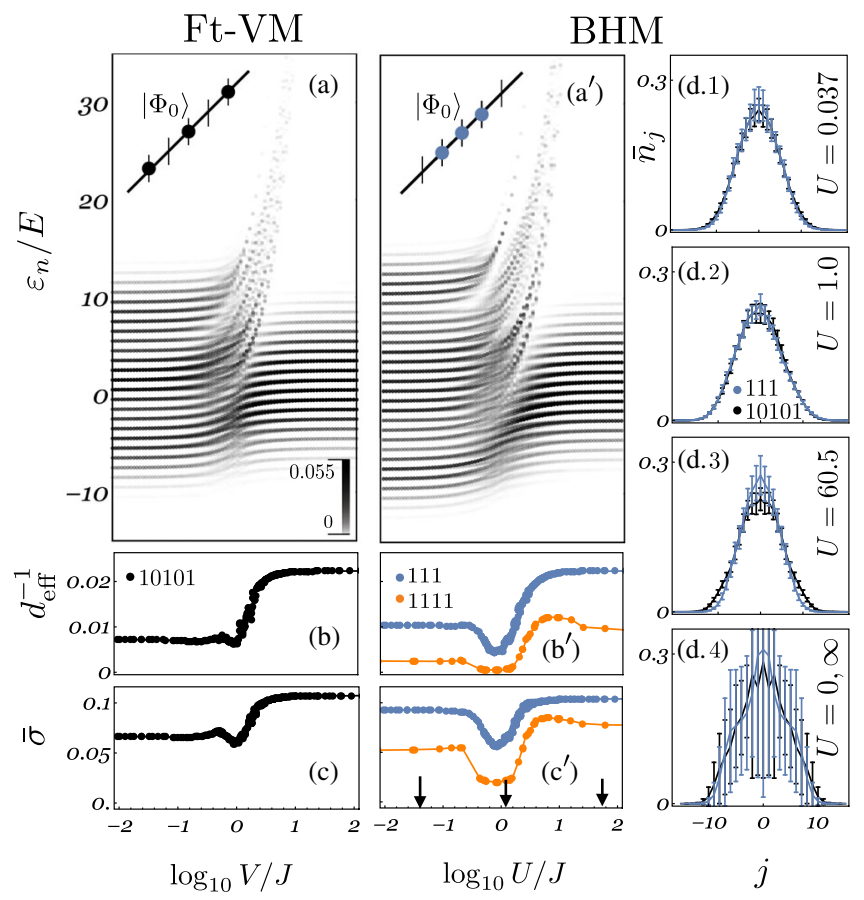

FIG. 1. (a),(a') Spectra of the Ft-VM and of the BHM as a function of the interaction strength. Line densities correspond to the overlap squared $\left|c_{\alpha}\right|^{2}$ of the initial state $\left|\Phi_{0}\right\rangle$, which is $|\ldots 0101010 \ldots\rangle$ for Ft-VM and $|\ldots 01110 \ldots\rangle$ for BHM, shown as insets. (b), (b') Inverse of effective dimension, $d_{\text {eff }}$ (c), (c') Strength $\bar{\sigma}$ of temporal fluctuations around the asymptotic longtime density average, for $N_{p}=3$ and 4. (d.1)-(d.4) Asymptotic average of the density profile $\bar{n}_{j}$ for two initial states with similar energies. Error bars depict fluctuations $\bar{\sigma}_{j}$ at each site. Three finite values of $U$ [marked by arrows in $\left.\left(\mathrm{c}^{\prime}\right)\right]$ and $U=0, \infty$ are used. 
eigenstates, and hence also the cloud dynamics, are exponentially localized.

The effective dimensionality of the Hilbert space spanned by the initial state is $d_{\text {eff }}=\left(\sum_{\alpha}\left|c_{\alpha}\right|^{4}\right)^{-1}$ [93,94], shown in Figs. 1(b) and $1\left(b^{\prime}\right)$. This quantity is larger for intermediate interactions than near the free points (small or large $U, V$ ). $d_{\text {eff }}$ decreases algebraically with $E$ and increases algebraically with particle number $N_{p}$.

We now analyze the longtime asymptotic behavior of the cloud dynamics in light of these spectral properties. We define the time averaged density $\bar{n}_{j}=\lim _{T \rightarrow \infty} T^{-1} \int_{0}^{T} d t n_{j}(t)$, with $n_{j}(t)=\left\langle a_{j}^{\dagger}(t) a_{j}(t)\right\rangle$ the site occupancy, and $\bar{\sigma}_{j}^{2}=$ $\lim _{T \rightarrow \infty} T^{-1} \int_{0}^{T} d t\left[n_{j}(t)-\bar{n}_{j}\right]^{2}$, which quantifies the temporal deviations around the average. For a system with a nondegenerate spectrum, these quantities are given by their diagonal ensemble [12] values $\bar{n}_{j}=\sum_{\alpha}\left|c_{\alpha}\right|^{2}\left\langle\alpha\left|n_{i}\right| \alpha\right\rangle$ and $\bar{\sigma}_{j}^{2}=\sum_{\alpha \neq \alpha^{\prime}}\left|c_{\alpha}\right|^{2}\left|c_{\alpha^{\prime}}\right|^{2}\left|\left\langle\alpha\left|n_{i}\right| \alpha^{\prime}\right\rangle\right|^{2}$. Some representative density profiles and $\bar{\sigma}=\left(\sum_{j} \sigma_{j}^{2}\right)^{1 / 2}$ for different values of $U$ and $V$ are depicted in Figs. 1(c), 1(c'), and 1(d).

For systems fulfilling the eigenstate thermalisation hypothesis (ETH) [12,95,96], temporal fluctuations of local observables are strongly suppressed, decreasing exponentially with system size $[97,98]$. In contrast, for integrable models, ETH does not hold: The decrease is merely polynomial. In the present case the system does not fulfill ETH trivially - there are an infinite number of eigenstates with the same energy but a vanishing overlap with the initial state. Moreover, as all eigenstates are localized throughout the spectrum, the system is many-bodylocalized.

Nonetheless, away from the free points, equilibration may still arise for sufficiently large $N_{p}$, i.e., large $d_{\text {eff }}$, in the sense that (A) different trapped initial states with roughly the same energy yield the same $\bar{n}_{i}$ profile and that (B) longtime deviations from the average are suppressed as $\bar{\sigma} \sim d_{\mathrm{eff}}^{-1 / 2}$ [97,98]. Property (A) is supported in Fig. 1(d). Comparison of Figs. 1(b) and 1(b') and Figs. 1(c) and 1(c') supports property (B) (as $d_{\text {eff }}^{-1}$ and $\bar{\sigma}$ are qualitatively similar). Also, $\bar{\sigma}$ substantially decreases with $N_{p}$ [Figs. $1\left(\mathrm{~b}^{\prime}\right)$ and $1\left(\mathrm{c}^{\prime}\right)$ ]. This supports an equilibration scenario for both fermionic and bosonic systems away from $U, V=0$ and $U, V=\infty$. At these special values the system becomes integrable and the limits $U, V \rightarrow 0, \infty$ and $t \rightarrow \infty$ do not commute. At these points $\bar{\sigma}$ is much larger and decreases much slower with particle number.

Dynamics near free points.-Figure 2 shows some BHM time evolutions at finite interaction values near the free points $U=0, \infty$. The center of mass $\langle x\rangle_{t}=\left(\sum_{j} n_{j}(t) j\right) / N_{p}$ and the width $\sigma_{t}=\left[\sum_{j}\left(j-\langle x\rangle_{t}\right)^{2} n_{j}(t) / N_{p}\right]^{1 / 2}$ of the cloud both generically show a "collapse and revival" or beating behavior. Other cloud characteristics (skewness or kurtosis) show the same effect [92]. To what extent the phenomenon is visible varies with the initial state and the quantity observed,
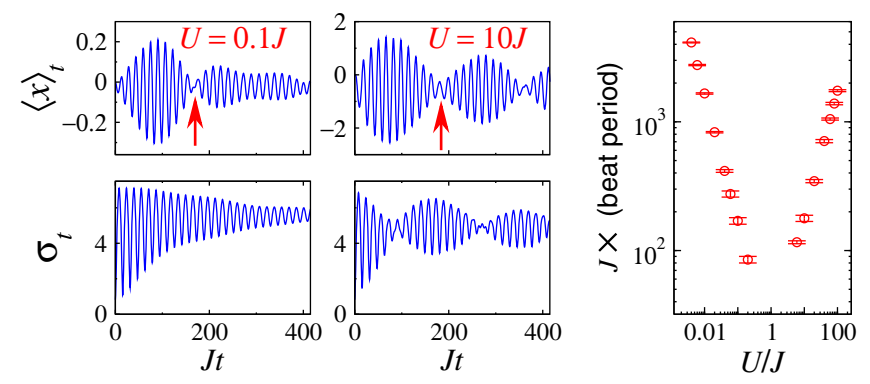

FIG. 2. Beating or relaxation of Bloch oscillations near the free points. BHM, 3 particles in 49 sites, $E=0.2 J$, product initial state $(\ldots 01110 \ldots)$. Arrows indicate beat periods.

but generically for $U / J$ not too close to 1 , a beat is visible. The beat period is seen to have clear linear dependences, $\propto U^{-1}$ at small $U / J$ and $\propto U$ at large $U / J$, on the interaction. The behavior at small $U / J$ has recently been observed experimentally [39]. We have found the same behavior in the fermionic case as a function of $V$ [92].

This remarkably simple dependence can be explained using the many-body spectrum. At the free points, this spectrum is exactly equally spaced (steps of $E$ ) and highly degenerate. As one moves away from these simple points, the degeneracy is lifted, so that the frequencies available for the dynamics are a range of values around $E$, the range being small compared to $E$. This explains the beat behavior. A perturbative argument yields an energy level splitting of the order of $V^{\nu}$ or $U^{\nu}$ with $\nu= \pm 1$ for weak or strong interactions. The splitting scale provides the beat frequency.

Spectral considerations also explain why there is rapid relaxation behavior without beats in the $U, V \sim J$ regime. In this regime, the eigenstates mix, destroying the ladder structure, and the chaotic structure of the spectrum leads to relaxation, as we have analyzed above. The present study in terms of the spectrum thus explains the results of the experiments of Ref. [39].

Cloud dynamics at free'points.-In contrast with the equilibration seen for moderate interactions, at $U, V=0$, $\infty$ there are perfectly periodic oscillations. The long-term state is not equilibrated and has strong dependence on the initial condition.

Figure 3 shows time evolution for the Jordan-Wignerrelated cases $V=0$ and $U \rightarrow \infty$, respectively labeled by $F$ or $B$. The cases of an initially spread-out and narrow cloud (small and large $\tilde{\rho}$ ) are shown (top and bottom). The density plots show the evolution of the density $n_{j}(t)$ (identical for $F$ and $B$ ) and of the momentum occupation number $\tilde{n}_{F}(k, t)$ and $\tilde{n}_{B}(k, t)$. For the bosonic system we also compute the occupation numbers of the natural orbitals $\lambda_{n}(t)[99,100]$ (with $\lambda_{0} \geq \lambda_{1} \geq \cdots$ ), defined as the eigenvalues of the single-particle density matrix $\rho_{B i, j}(t)=\left\langle b_{i}^{\dagger}(t) b_{j}(t)\right\rangle$. A macroscopic occupation (i.e., a $\lambda_{i}$ of order $\sqrt{N_{p}}$ ) corresponds to quasicondensation.

The density profile $n(x, t)$ displays qualitatively different dynamics for small and large $\tilde{\rho}$ : Bloch oscillations consist of 


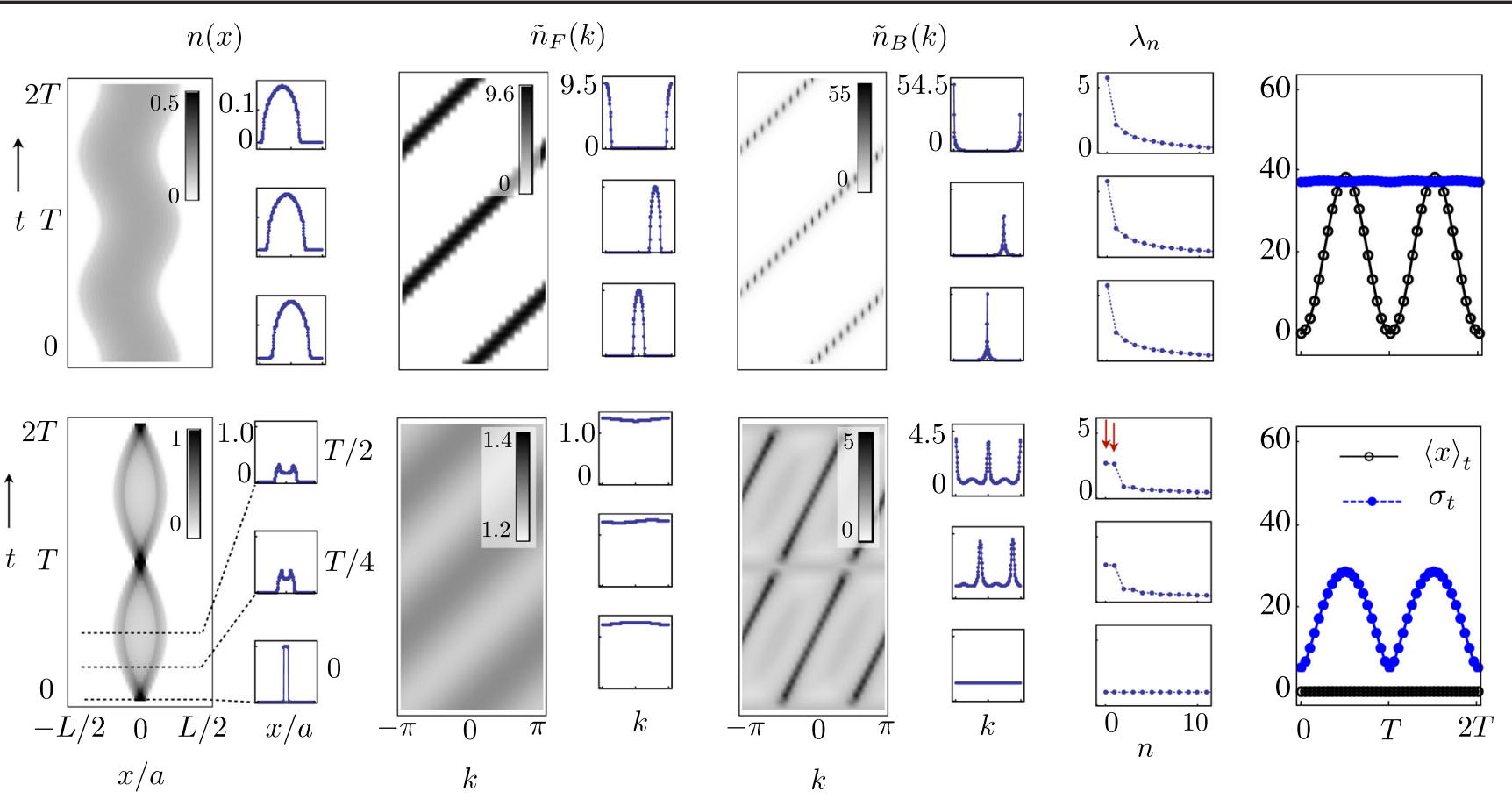

FIG. 3. Time evolution of $n(x), \tilde{n}_{F}(k)=\sum_{x}(1 / \sqrt{L}) \sum_{y} \rho_{y+x, y}^{F} e^{-i k x}, \tilde{n}_{B}(k)=\sum_{x}(1 / \sqrt{L}) \sum_{y} \rho_{y+x, y}^{B} e^{-i k x}$, and $\lambda_{n}$ computed for $L=250, N_{p}=20, E=0.05 \mathrm{~J}$. Upper (lower) row: $\tilde{\rho}=0.1(\tilde{\rho}=10)$. The insets display the profiles of the different quantities for $t=T$, $t=T / 4$, and $t=T / 2$, where $T=2 \pi / E$ is the oscillation period. Right-hand panels show evolution of the center of mass and width of the cloud.

mainly position oscillations for $\tilde{\rho} \ll 1$ and mainly width oscillations for $\tilde{\rho} \gg 1$. For large $\tilde{\rho}$, the shape of the initially localized cloud changes considerably within a period, the shape becoming double peaked when the cloud widens. The oscillation amplitude of the center of mass $\langle x\rangle_{t}$ is large for $\tilde{\rho} \ll 1$ and small for $\tilde{\rho} \gg 1$. The cloud width $\sigma_{t}$ shows the opposite behavior (Fig. 3, far right.) This distinction is analogous to that observed in single-particle Bloch oscillations [15]. Additional shape dynamics appear at intermediate $\tilde{\rho}$ - the cloud becomes strongly skewed once every period [92]. The amplitude of skewness oscillations is nonmonotonic as a function of $\tilde{\rho}$, being zero for $\tilde{\rho} \rightarrow 0$ and $\tilde{\rho} \rightarrow \infty$ and nonzero for intermediate $\tilde{\rho}$. [Contrast the amplitude of position (width) oscillations, which decreases (increases) monotonically with $\tilde{\rho}$.] The exact behavior of the skewness oscillation amplitude as a function of $\tilde{\rho}$ depends on the particle number $N_{p}$ in a complicated manner for small $N_{p}$, but for $N_{p} \gtrsim 20$ there is a unique (negative) minimum that shifts to larger $\tilde{\rho}$ with increasing $N_{p}$ [92].

For any Gaussian initial state, the subsequent cloud dynamics (time evolution of moments) can be obtained analytically as a function of the initial moments of correlators [92]. The center of mass has purely sinusoidal oscillations, $\langle x\rangle_{t}=-(2 J / E) \sin ^{2}(t E / 2) \mu_{1}$. The width dynamics is more complicated: $\sigma_{t}^{2}-\sigma_{t=0}^{2}=-\left(4 J^{2} / E^{2}\right) \sin ^{4}(t E / 2) \mu_{1}^{2}+$ $2(J / E)^{2} \sin ^{2}[(E / 2) t]\left[1-\cos (E t) \mu_{2}\right]$. Here, $\mu_{a}=\left(1 / 2 N_{p}\right) \times$ $\sum_{y}\left\langle c_{y}^{\dagger} c_{y-a}+c_{y-a}^{\dagger} c_{y}\right\rangle_{t=0}$. (The behavior of $\mu_{1}$ and $\mu_{2}$ as functions of $N_{p}$ and $\tilde{\rho}$ is described in Ref. [92].) This allows us to compute the amplitudes of oscillation of the moments, e.g., $\Delta x=\max _{t}\langle x\rangle_{t}-\min _{t}\langle x\rangle_{t}$, and $\Delta \sigma^{2}=\max _{t} \sigma_{t}^{2}-\min _{t} \sigma_{t}^{2}$ as a function of $\tilde{\rho}$ for different $N_{p}$. The position oscillation amplitude is $\Delta x=2 J / E$ for $\tilde{\rho} \rightarrow 0$, and at large $\tilde{\rho}$ decreases as $\Delta x \propto J /(E \tilde{\rho})$ for $N_{p}>1$ [92]. Conversely, $\Delta \sigma^{2}$ increases from zero to $2(J / E)^{2}$ as $\tilde{\rho}$ is increased $\left(N_{p}>1\right)$ [92].

The momentum distribution of the fermionic system $(F)$ has simple time evolution: $n_{F}(k, t)=n_{F}[k-(2 \pi / T) t, 0]$, reminiscent of single-particle Bloch oscillations [92]. For $B$, the momentum distribution $n_{B}(k, t)$ has similar behavior for small $\tilde{\rho}$, but it is now a sharply peaked distribution that traverses the Brillouin zone periodically, signaling quasicondensation in the initial state that survives during the oscillations. The natural orbital occupancy accordingly shows a dominant eigenvalue that stays dominant throughout the evolution. The large $\tilde{\rho}$ behavior is more intricate. Although the condensate is initially noncondensed, two well-defined coherence peaks appear. Remarkably, they disappear periodically for a short fraction of the period when returning to the initial state. The set $\left\{\lambda_{i}\right\}$ now has two dominant occupancies, $\lambda_{0} \gg \lambda_{n>0}$, signaling a fragmented condensate that is dynamically generated [75,101] and persists for almost all times within each period.

Discussion.-We have presented a thorough study of many-body Bloch dynamics in two standard lattice models in one dimension, one fermionic and one bosonic. A main result is that generic many-body systems under a tilt potential have a dichotomic nature, possessing both ETH 
and many-body-localized features. Although their eigenstates are exponentially localized, and an initially trapped cloud has finite overlap only with a zero-measure set of eigenstates within the relevant energy window, the longtime dynamics yield a thermalized state within a Hilbert space of effective dimension $d_{\text {eff }}$ which increases with the number of particles $N_{p}$.

The approach to the thermalized state can be seen as the destruction of the many-body Bloch oscillations which are present at the integrable (free) limits, both for weak and strong coupling. We show that the relevant timescale grows as $U\left(U^{-1}\right)$ or $V\left(V^{-1}\right)$ away from the weak (strong) integrable limit. At the free limits we present several striking features of the cloud dynamics, including a dynamical generation (and periodic disappearance) of fragmented condensation for strong initial trapping.

M. H. thanks H. C. Nägerl for useful discussions. P. R. acknowledges support by FCT through the Investigador FCT Contract No. IF/00347/2014 and Grant No. UID/ CTM/04540/2019.

*ribeiro.pedro@gmail.com

[1] O. Bohigas and H. A. Weidenmuller, Annu. Rev. Nucl. Part. Sci. 38, 421 (1988).

[2] V. Zelevinsky, Annu. Rev. Nucl. Part. Sci. 46, 237 (1996).

[3] H. A. Weidenmüller and G. E. Mitchell, Rev. Mod. Phys. 81, 539 (2009).

[4] I. Bloch, J. Dalibard, and W. Zwerger, Rev. Mod. Phys. 80, 885 (2008).

[5] O. Morsch and M. Oberthaler, Rev. Mod. Phys. 78, 179 (2006).

[6] T. Kinoshita, T. Wenger, and D. S. Weiss, Nature (London) 440, 900 (2006).

[7] M. Greiner, O. Mandel, T. W. Hänsch, and I. Bloch, Nature (London) 419, 51 (2002).

[8] L. D'Alessio, Y. Kafri, A. Polkovnikov, and M. Rigol, Adv. Phys. 65, 239 (2016).

[9] J. Eisert, M. Friesdorf, and C. Gogolin, Nat. Phys. 11, 124 (2015).

[10] A. Polkovnikov, K. Sengupta, A. Silva, and M. Vengalattore, Rev. Mod. Phys. 83, 863 (2011).

[11] J. Dziarmaga, Adv. Phys. 59, 1063 (2010).

[12] M. Rigol, V. Dunjko, and M. Olshanii, Nature (London) 452, 854 (2008).

[13] F. Bloch, Z. Phys. 52, 555 (1929).

[14] C. Zener, Proc. R. Soc. A 145, 523 (1934).

[15] T. Hartmann, F. Keck, H. J. Korsch, and S. Mossmann, New J. Phys. 6, 2 (2004).

[16] Q. Thommen, J. C. Garreau, and V. Zehnlé, J. Opt. B 6, 301 (2004).

[17] C. Waschke, H. G. Roskos, R. Schwedler, K. Leo, H. Kurz, and K. Köhler, Phys. Rev. Lett. 70, 3319 (1993).

[18] V. G. Lyssenko, G. Valušis, F. Löser, T. Hasche, K. Leo, M. M. Dignam, and K. Köhler, Phys. Rev. Lett. 79, 301 (1997).
[19] M. Ben Dahan, E. Peik, J. Reichel, Y. Castin, and C. Salomon, Phys. Rev. Lett. 76, 4508 (1996).

[20] Q. Niu, X.-G. Zhao, G. A. Georgakis, and M. G. Raizen, Phys. Rev. Lett. 76, 4504 (1996).

[21] M. Raizen, C. Salomon, and Q. Niu, Phys. Today 50, No. 7, 30 (1997).

[22] B. P. Anderson and M. A. Kasevich, Science 282, 1686 (1998).

[23] R. Morandotti, U. Peschel, J. S. Aitchison, H. S. Eisenberg, and Y. Silberberg, Phys. Rev. Lett. 83, 4756 (1999).

[24] T. Pertsch, P. Dannberg, W. Elflein, A. Bräuer, and F. Lederer, Phys. Rev. Lett. 83, 4752 (1999).

[25] R. Sapienza, P. Costantino, D. Wiersma, M. Ghulinyan, C. J. Oton, and L. Pavesi, Phys. Rev. Lett. 91, 263902 (2003).

[26] O. Morsch, J. H. Müller, M. Cristiani, D. Ciampini, and E. Arimondo, Phys. Rev. Lett. 87, 140402 (2001).

[27] M. Cristiani, O. Morsch, J. H. Müller, D. Ciampini, and E. Arimondo, Phys. Rev. A 65, 063612 (2002).

[28] G. Roati, E. de Mirandes, F. Ferlaino, H. Ott, G. Modugno, and M. Inguscio, Phys. Rev. Lett. 92, 230402 (2004).

[29] R. Battesti, P. Cladé, S. Guellati-Khélifa, C. Schwob, B. Grémaud, F. Nez, L. Julien, and F. Biraben, Phys. Rev. Lett. 92, 253001 (2004).

[30] G. Ferrari, N. Poli, F. Sorrentino, and G. M. Tino, Phys. Rev. Lett. 97, 060402 (2006).

[31] P. Cladé, E. de Mirandes, M. Cadoret, S. Guellati-Khélifa, C. Schwob, F. Nez, L. Julien, and F. Biraben, Phys. Rev. A 74, 052109 (2006).

[32] S. Drenkelforth, G. Kleine Büning, J. Will, T. Schulte, N. Murray, W. Ertmer, L. Santos, and J. J. Arlt, New J. Phys. 10, 045027 (2008).

[33] M. Gustavsson, E. Haller, M. J. Mark, J. G. Danzl, G. Rojas-Kopeinig, and H.-C. Nägerl, Phys. Rev. Lett. 100, 080404 (2008).

[34] E. Haller, R. Hart, M. J. Mark, J. G. Danzl, L. Reichsöllner, and H.-C. Nägerl, Phys. Rev. Lett. 104, 200403 (2010).

[35] N. Poli, F.-Y. Wang, M. G. Tarallo, A. Alberti, M. Prevedelli, and G. M. Tino, Phys. Rev. Lett. 106, 038501 (2011).

[36] L. Tarruell, D. Greif, T. Uehlinger, G. Jotzu, and T. Esslinger, Nature (London) 483, 302 (2012).

[37] M. G. Tarallo, A. Alberti, N. Poli, M. L. Chiofalo, F.-Y. Wang, and G. M. Tino, Phys. Rev. A 86, 033615 (2012).

[38] M. Genske, W. Alt, A. Steffen, A. H. Werner, R. F. Werner, D. Meschede, and A. Alberti, Phys. Rev. Lett. 110, 190601 (2013).

[39] F. Meinert, M. J. Mark, E. Kirilov, K. Lauber, P. Weinmann, M. Gröbner, and H.-C. Nägerl, Phys. Rev. Lett. 112, 193003 (2014).

[40] P. M. Preiss, R. Ma, M. E. Tai, A. Lukin, M. Rispoli, P. Zupancic, Y. Lahini, R. Islam, and M. Greiner, Science 347, 1229 (2015).

[41] H. Keßler, J. Klinder, B. P. Venkatesh, C. Georges, and A. Hemmerich, New J. Phys. 18, 102001 (2016).

[42] C. Georges, J. Vargas, H. Keßler, J. Klinder, and A. Hemmerich, Phys. Rev. A 96, 063615 (2017).

[43] Z. A. Geiger, K. M. Fujiwara, K. Singh, R. Senaratne, S. V. Rajagopal, M. Lipatov, T. Shimasaki, R. Driben, V. V. Konotop, T. Meier, and D. M. Weld, Phys. Rev. Lett. 120, 213201 (2018). 
[44] A. R. Kolovsky, A. V. Ponomarev, and H. J. Korsch, Phys. Rev. A 66, 053405 (2002).

[45] B. M. Breid, D. Witthaut, and H. J. Korsch, New J. Phys. 8, 110 (2006).

[46] B. M. Breid, D. Witthaut, and H. J. Korsch, New J. Phys. 9, 62 (2007).

[47] Q. Thommen, J. C. Garreau, and V. Zehnlé, Am. J. Phys. 72, 1017 (2004).

[48] S. Walter, D. Schneble, and A. C. Durst, Phys. Rev. A 81, 033623 (2010).

[49] S. Longhi, Phys. Rev. B 81, 195118 (2010).

[50] M. Collura, H. Aufderheide, G. Roux, and D. Karevski, Phys. Rev. A 86, 013615 (2012).

[51] A. R. Kolovsky and E. N. Bulgakov, Phys. Rev. A 87, 033602 (2013).

[52] S. Roy, A. G. Grushin, R. Moessner, and M. Haque, Phys. Rev. A 92, 063626 (2015).

[53] R. Khomeriki and S. Flach, Phys. Rev. Lett. 116, 245301 (2016).

[54] Y. V. Kartashov, V. V. Konotop, D. A. Zezyulin, and L. Torner, Phys. Rev. Lett. 117, 215301 (2016).

[55] S. Longhi, Int. J. Mod. Phys. B 30, 1650189 (2016).

[56] Y. Zheng, S. Feng, and S.-J. Yang, Phys. Rev. A 96, 063613 (2017).

[57] W. S. Dias, E. M. Nascimento, M. L. Lyra, and F. A. B. F. de Moura, Phys. Rev. B 76, 155124 (2007).

[58] D. O. Krimer, R. Khomeriki, and S. Flach, Phys. Rev. E 80, 036201 (2009).

[59] W. S. Dias, E. M. Nascimento, M. L. Lyra, and F. A. B. F. de Moura, Phys. Rev. B 81, 045116 (2010).

[60] S. Longhi and G. Della Valle, Phys. Rev. B 85, 165144 (2012).

[61] D. Wiater, T. Sowiński, and J. Zakrzewski, Phys. Rev. A 96, 043629 (2017).

[62] A. Buchleitner and A. R. Kolovsky, Phys. Rev. Lett. 91, 253002 (2003).

[63] A. R. Kolovsky, Phys. Rev. Lett. 90, 213002 (2003).

[64] A. R. Kolovsky and H. J. Korsch, Int. J. Mod. Phys. B 18, 1235 (2004).

[65] T. Schulte, S. Drenkelforth, G. K. Büning, W. Ertmer, J. Arlt, M. Lewenstein, and L. Santos, Phys. Rev. A 77, 023610 (2008).

[66] M. Salerno, V. V. Konotop, and Y. V. Bludov, Phys. Rev. Lett. 101, 030405 (2008).

[67] M. Lepers, V. Zehnlé, and J. C. Garreau, Phys. Rev. Lett. 101, 144103 (2008).

[68] A. R. Kolovsky, H. J. Korsch, and E.-M. Graefe, Phys. Rev. A 80, 023617 (2009).

[69] A. R. Kolovsky, E. A. Gómez, and H. J. Korsch, Phys. Rev. A 81, 025603 (2010).

[70] M. Mierzejewski and P. Prelovšek, Phys. Rev. Lett. 105, 186405 (2010).

[71] M. Eckstein, T. Oka, and P. Werner, Phys. Rev. Lett. 105, 146404 (2010).

[72] M. Eckstein and P. Werner, Phys. Rev. Lett. 107, 186406 (2011).

[73] D. Witthaut, F. Trimborn, V. Kegel, and H. J. Korsch, Phys. Rev. A 83, 013609 (2011).
[74] C. P. Rubbo, S. R. Manmana, B. M. Peden, M. J. Holland, and A. M. Rey, Phys. Rev. A 84, 033638 (2011).

[75] X. Cai, S. Chen, and Y. Wang, Phys. Rev. A 84, 033605 (2011).

[76] S. Mandt, A. Rapp, and A. Rosch, Phys. Rev. Lett. 106, 250602 (2011).

[77] C. Gaul, E. Díaz, R. P. A. Lima, F. Domínguez-Adame, and C. A. Müller, Phys. Rev. A 84, 053627 (2011).

[78] S. Longhi, Phys. Rev. B 86, 075144 (2012).

[79] J. Carrasquilla, S. R. Manmana, and M. Rigol, Phys. Rev. A 87, 043606 (2013).

[80] S. Mandt, Phys. Rev. A 90, 053624 (2014).

[81] K. W. Mahmud, L. Jiang, E. Tiesinga, and P. R. Johnson, Phys. Rev. A 89, 023606 (2014).

[82] R. Driben, V. Konotop, T. Meier, and A. Yulin, Sci. Rep. 7, 3194 (2017).

[83] U. Gavish and Y. Castin, Phys. Rev. Lett. 95, 020401 (2005).

[84] N. Y. Yao, C. R. Laumann, J. I. Cirac, M. D. Lukin, and J. E. Moore, Phys. Rev. Lett. 117, 240601 (2016).

[85] M. Schiulaz, A. Silva, and M. Müller, Phys. Rev. B 91, 184202 (2015).

[86] A. E. Antipov, Y. Javanmard, P. Ribeiro, and S. Kirchner, Phys. Rev. Lett. 117, 146601 (2016).

[87] A. Smith, J. Knolle, D. L. Kovrizhin, and R. Moessner, Phys. Rev. Lett. 118, 266601 (2017).

[88] R. Mondaini and Z. Cai, Phys. Rev. B 96, 035153 (2017).

[89] M. Schulz, C. A. Hooley, R. Moessner, and F. Pollmann, Phys. Rev. Lett. 122, 040606 (2019).

[90] M. Rigol and A. Muramatsu, Phys. Rev. A 69, 053612 (2004).

[91] M. Rigol and A. Muramatsu, Phys. Rev. A 70, 043627 (2004).

[92] See Supplemental Material at http://link.aps.org/ supplemental/10.1103/PhysRevLett.124.110603 where we describe the four non-interacting ("free") points in the two one-dimensional models we have considered, we provide a number of exact results, we discuss the beating behavior and provide further numerical data, and discuss some aspects of the many-body spectrum, including levelspacing statistics.

[93] S. Popescu, A. J. Short, and A. Winter, Nat. Phys. 2, 754 (2006).

[94] N. Linden, S. Popescu, A. J. Short, and A. Winter, Phys. Rev. E 79, 061103 (2009).

[95] J. M. Deutsch, Phys. Rev. A 43, 2046 (1991).

[96] M. Srednicki, Phys. Rev. E 50, 888 (1994).

[97] P. R. Zangara, A. D. Dente, E. J. Torres-Herrera, H. M. Pastawski, A. Iucci, and L. F. Santos, Phys. Rev. E 88, 032913 (2013).

[98] W. Beugeling, R. Moessner, and M. Haque, Phys. Rev. E 91, 012144 (2015).

[99] O. Penrose and L. Onsager, Phys. Rev. 104, 576 (1956).

[100] A. Leggett, Rev. Mod. Phys. 73, 307 (2001).

[101] M. Rigol and A. Muramatsu, Phys. Rev. Lett. 93, 230404 (2004). 